\title{
Un sogno umanistico figurato: Delfilo
}

\author{
Marcello Ciccuto
}

Università di Pisa

\begin{abstract}
In questo saggio ho studiato un poemetto illustrato del XVI secolo, il Somnium Delphili, opera di un nobile di Piacenza, Marco Antonio Ceresa, che raccontò in esso la storia di un suo amore contrastato. Le varie situazioni narrative vengono utilizzate quali pretesti verbali per la raffigurazione di sogni o di transferts mitografici, che trasformano quella vicenda biografica in un percorso fra simboli antichi: interessante episodio di passaggio del mondo antiquario rinascimentale verso le codificazioni emblematiche dell'età più matura.
\end{abstract}

Parole chiave: Delfilo, storie d'amore rinascimentali, mitografie, sogni figurati, simboli iconografici.

\section{Abstract}

This essay studies an illustrated short poem of the XVI ${ }^{\text {th }}$ century, the Somnium Delphili, written and probably illustrated by a Piacenza nobleman, Marco Antonio Ceresa, who told the tale of a troubled love affair. The various narrative situations are here used as verbal pretexts for the representation of dreams or mythographic transfers, which are used to transform these biographical vicissitudes into a journey among ancient symbols - that is an interesting episode of a passage from the ancient Renaissance world to a more mature emblematic codification.

Key words: Delphilus, Renaissance love stories, mythographies, iconized dreams, iconographical symbols.

In un codice oggi ambrosiano in scrittura umanistica libraria (C 20 inf.) è conservato in copia unica un poemetto cinquecentesco illustrato, in terza rima, il Somnium Delphili, preceduto da una prosa latina che ne avvisa alcuni risvolti tematici e d'occasione. Riconosciuto in un primo momento da padre Giovanni Pozzi come opera - nel codice comunque non autografa - del Francesco Colonna autore dell' Hypnerotomachia Poliphili, ${ }^{1}$ il poemetto si ebbe in

1. Lo studio classico in argomento è appunto quello di Maria Teresa Casella e Giovanni POZZI, Francesco Colonna. Biografia e opere, II, Padova: Antenore, 1959, p. 161 e sgg., segui- 
seguito le attenzioni di una ricerca specifica di Maria Corti la quale, finalmente adeguando la lettura filologica agli esiti dell'interpretazione iconografica, riusciva a determinare le radici piacentine dell'autore-protagonista nonché i numerosi agganci storico-biografici della partitura figurata che spingevano a identificarlo con quel Marco Antonio Ceresa, nobile piacentino (1488-1526), che l'infiammato ciliegio dipinto alla carta $29 \mathrm{r}$ ci conferma preso a una certa altezza della sua parabola esistenziale dagli spasimi del fuoco d'amore. ${ }^{2}$ Invero anche la raffigurazione del fortificato castello di Momeliano (c. 17r) pare intenzionalmente rievocare una storia di amore contrastato del Ceresa, estraneo e precedente a quello legittimo per una Lodovica Caccia pur condotta a nozze combinate nel 1508 (nozze di cui la miniatura di p. 51r evoca le ragioni grettamente economiche nella forma delle gambe flesse di fronte a un mucchietto di ducati): ${ }^{3}$ perché fu proprio in quella dimora che il Ceresa-Delfilo, nel corso della peste che afflisse Pavia nei primissimi anni del ' 500 , ospitò la fanciulla di cui si invaghì; salvo poi perderla nel tumulto della storia e reincontrarla - una volta sposato- per far scaturire le antiche fiamme oramai non più avvertite né condivise dall'ex-amante, anzi letteralmente "freddate» con atteggiamento in conclusione scostante di fin de non-recevoir.

L'elemento impegnato testualmente e figurativamente nella tematica del sogno pertiene, in mezzo a tanta effusione lirica di taglio descrittivo, proprio all'area nella quale il poeta si dedica a rammemorare in visione la bellezza dell'amata in minutissimi particolari: dal testo parte invariabilmente l'allusione a un'immagine, a una serie di immagini che hanno il compito di portare l'accadimento entro una zona di pura significazione simbolica e appunto oniri$\mathrm{ca}$, spostata rispetto al piano dei più semplici referenti fattuali e autonomamente significante. Già le prime carte figurate del codice impostano detta resa simbolica entro la griglia semantica in chiave oppositiva della fiamma d'amore contrapposta al gelo repulsivo della donna. Ad esempio la raffigurazione d'esordio della fucina di Vulcano, c. 1v, spinge, nella ben strutturata combinazione fra testi in epigrafe e azione narrativa, a comunicare (nelle parole di padre Pozzi) «da una parte l'ardore dell'innamorato protagonista (frecce e fuoco), dall'altra la renitenza e freddezza (corazze e fonti) della protagonista. Ma l'antitesi fuoco-acqua ritorna, come un «leit-motiv» in più luoghi dell'elegia, specialmente ai vv. 28$30,121-26,169,1675-98,2157 » .{ }^{4}$ Dalla stessa descrizione in parole offerta

to, oltreché dal saggio di Maria CORTI citato poco più avanti, dalle importanti acquisizioni documentarie di Giuseppe FIORI, "Il poeta piacentino Marco Antonio Ceresa ed il suo castello di Momeliano", Bollettino storico piacentino, 1986, p. 255-264.

2. Cfr. Maria CORTI, «Da un convento veneto a un castello piacentino (L'autore del «Delfilo» non è Francesco Colonna)», Giornale storico della letteratura italiana, CXXXVIII, 1961, p. 161-195.

3. La corretta interpretazione di alcune delle illustrazioni del codice si deve, come pure in questo caso, al recente intervento di Alessandro RoveTTA, «Il Somnium Delphili dell'Ambrosiana. Un contributo all'ermetismo figurativo di primo Cinquecento", Rivista di storia della miniatura, 1-2, 1996-1997, p. 61-68.

4. Cfr. dunque ancora Maria Teresa CASElla e Giovanni Pozzi, Francesco Colonna, cit., p. 164. 
da Pozzi per questa illustrazione si capisce assai bene la stretta relazione che intercorre fra le parole e le immagini — anche a livello di resa "commentativa» in forma di epigrafe - sulla via di una sorta di rappresentazione del sogno dell'autore-protagonista espansa nello spazio; sì che giova riportare qui di seguito la suddetta descrizione:

A sinistra, una palma di forma alquanto approssimativa: sopra la chioma sta scritto in rosso: "Palma». Sull'albero, appese ad un nastro, tre frecce ed una tavoletta colla scritta: "Quis evadet». A destra un alloro, pure con l'indicazione in rosso: "Lauro", cui sono sospese in modo identico frecce e tavoletta, colla scritta «Nemo». [...] In cima alla roccia che si alza sopra la fucina Cupido bendato tira una freccia contro una stella; un cartiglio che cinge il corpo del dio dice: «Nec si Jovis arma resistant». [...] A destra dietro la roccia appare un corso d'acqua: una figura inginocchiata sulla riva alza la mano destra impugnando una freccia; dentro l'acqua sta scritto «arbonensis lacus» (cfr. vv. 115-17). ${ }^{5}$

Né meno interessante l'illustrazione posta alla c. 2r, susseguente anche narrativamente alla prima, dove

Delle ninfe martellano delle corazze, entro una fucina in una grotta, in cui non c'è però fuoco. Due piante stanno ai lati del disegno: a destra un olivo, a sinistra un agnocasto (in rosso sta scritto rispettivamente "Oliva» ed "Agnochasto»). Come là le frecce, qui sono appese delle corazze, sulle quali stanno scritte le seguenti indicazioni: dalla parte dell'olivo: «Sollicitudini», dalla parte dell'agnocasto «Dive lacunarum». Due cartelli identici a quelli della precedente miniatura, legati ai nastri che reggono le corazze, dicono nello stesso ordine: «Indomabile hoc»; "Quis evadet?». In basso un fiume attraversa l'illustrazione: nell'acqua è scritto: "Cydnus». Dalla roccia che sovrasta la fucina sgorgano, qua e là, due zampilli: a sinistra sta l'indicazione "Derce», a destra "Neme». Una ninfa, inginocchiata in riva al Cidno, vi tuffa una corazza: un motto scritto direttamente sul disegno, non chiuso in un cartiglio, dice: «Feriat tandem ferus ille Cupido». 6

La protagonista può insomma comparire a c. $8 \mathrm{v}$, in un'illustrazione a piena pagina tutta per sé, con gli attributi oramai a lei consentanei di freddezza e indifferenza: galleggiante sopra uno stagno posto fra due spuntoni rocciosi, in atto di reggere con la destra in alto, sopra la sua testa, un cartiglio coi versi pieni degli appellativi seguenti:

Quic quid Phoebus habet mihi contulit ipse Cupido:

Tela, faces, Genitrix et decus et Charites:

Hinc utcumque liber nudo sub tegmine Nympha

Thespis et Erothea dicor et Eucaria

5. Ibid., p. 163.

6. Ibid., p. 163-164. E per l'identificazione delle due ninfe (che presiedono a fonti notoriamente gelide), di cui scrivono sia Marziale sia il Perotti nelle sue Cornucopiae, cfr. di nuovo ibid., p. 173 . 
Per converso, il Ceresa-innamorato figura in un'immagine ancora a piena pagina (c. 69r), vestito di rosso sullo sfondo di rocce dalle quali escono fiamme:

La figura indica con una mano in alto le fiamme, con l'altra verso il basso l'acqua: è di nuovo il tema acqua-fuoco delle miniature ai ff. $1 \mathrm{v}-2 \mathrm{r}$, chiarito dai versi latini che sotto riporterò. A destra s'inalza un albero: la solita scritta in rosso, dice: «Mirto». Sul tronco dell'albero è scritta la prima terzina del poemetto: chiaro il riferimento a queste parole della prosa latina: «elegia inscripta chamaemirsyne». Ma si noti che è una pura allusione di passaggio: essa non ha sviluppo nella narrazione in modo da divenire un episodio ben definito: non s'intende come il testo, da sé solo, avrebbe potuto suggerire al pittore l'inclusione di questo dettaglio. All'albero è appesa una tabella coi seguenti versi:
Diversae hoc quod mortis habet michi morte rependit.
Exuror flammis, nauphragor in lachrymas.
Unda Cupidineos ignes, incendia lymphas
Emoderant, duplici salvor ab interitu.
Hinc, utcumque libet, nudo sub tegmine pastor
Dacryrous dicor, dicor et Euripyros.?

Del resto al tema del fuoco rinviano tutti i cartigli che, sul verso delle carte da 9 a 67, hanno impresso il testo sacer est ignis credite lesis, mentre altre figurazioni circolano attorno alla simbologia ignea nella forma di una fiamma minacciata da un fiumiciattolo (c. 53r), di un fuoco tra nuvole (c. 36r) e di un altro isolato (c. 64r), oppure alla simbologia della frigidità acquatica con la ninfa Ciane immersa in una fontana (c. 10r) e della mano aperta, coperta da un getto d'acqua (c. 46r).

Fin qui, come ben si intende, nulla di particolarmente originale; a meno che non rivolgiamo lo sguardo alla prima carta decorata del testo lirico (9r), nella quale Rossi ha giustamente ravvisato echi depredisiani e, poco più avanti, indicazioni d'area sforzesca: vuoi nella figurazione dell'unicorno dell'iniziale Q[uella], vuoi nel divino occhio onniperspiciente del margine inferiore al centro "tra penne di pavone, simbolo dell'immortalità, e fiori». ${ }^{8}$ E proprio a questa vistosa simbologia onirico-visionaria che fa da contrasto l'inserimento di immagini di più scoperta vettorialità informativa, come le due bisce di c. 11 r o l'aquila di c. $27 \mathrm{r}$, e infine il richiamo del mito di Deucalione e Pirra relativo alle origini del protagonista (c. $14 \mathrm{r}$ e v. 1350) o l'immagine del castello in rovina forse equivalente a quello di Momeliano restaurato dai Ceresa (c. 63r). Vettorialità informativa che insomma si spegne presto, a fronte dell'impegno dell'autore sul versante di un autentico effetto di trasvaloramento della vicenda biografica sul piano dello straniamento onirico. Intanto si dovrebbe dire a questo riguardo dell'aggancio immaginativo operato dal Ceresa nei confronti

8. Alessandro ROVETTA, «Il Somnium Delphili», cit., p. 62. 
di una complessa concettosità geroglifica, perlopiù svincolata da esigenze di corrispondenza letterale col testo del poemetto e invece incaricata di ispessire il tenore di espansione significante delle idee-guida poste in essere dalla collaborazione testo-immagine. Sarà il caso della riflessione sul tempus fugit, presa più e più volte nel giro di figure quali il rocchetto di filo e i due occhi di c. 26r, la falce a c. 66r e varie altre; ${ }^{9}$ mentre accompagnato dalla didascalia Tempus troviamo a c. 33r un Giano bifronte che guarda coi due volti (uno giovane e l'altro vecchio e barbuto) a un sole raggiante e a un sole eclissato, recando scritto sulla mano aperta la cifra 366 corrispondente forse ai giorni di un anno bisestile. Parrebbe avviata su analogo percorso interpretativo anche la figurazione di Occasio-Fortuna che alla c. $13 \mathrm{r}$ inscena l'usuale simbiosi rinascimentale col corpo della Nemesis. ${ }^{10}$

Resta tuttavia in primo piano, e all'interno di questo programma illustrativo non proprio perfetto in tutte le sue componenti, la capacità ceresiana di assommare una serie di immagini simboliche sullo spazio della pagina —e il più delle volte senza concreti allacci alla dimensione testuale-, quasi a riprodurre nella struttura codicologica di superficie il distribuirsi sconnesso e incoerente delle immagini nello spazio allargato e pressoché non limitato del sogno. ${ }^{11}$ Si rilevi in tal senso quanto la mancata allusione testuale all'epigramma In Hermaphroditum, tradotto in greco da Poliziano, ${ }^{12}$ non osti alla libertà rappresentativa, quasi del tutto autonoma e già di sapore emblematico, che presiede alla raffigurazione del giovane trafitto dalla propria spada, appeso per i piedi a un albero e con la testa in acqua (c. 16r). Così del tutto analogo, e persino con minore sostegno da parte della componente verbale del poemetto, pare il caso della figura di Arpocrate, dio del silenzio, che occorre alla c. $35 \mathrm{r}$ nel bel mezzo di una serie di paragoni con le dita della fanciulla innominata; fino al secco referto della mano con pugnale nell'atto di uccidere una scrofa nera (c. $47 \mathrm{r}$ ), forse riferibile a un episodio del mito di Meleagro ma in ogni caso luogo di conferma di una assoluta autonomia significante di immagini che l'autore ha saputo estrarre da quell'area emiliana del pensiero ermetico e neoplatonico nella quale l'idea di una funzione «creativa» delle immagini aveva conosciuto una

9. Altre figure connesse alla tematica del Tempo sono quelle alla c. 52r (Ercole che incatena Cerbero), alla c. 54r (Crono che divora i figli), l'immagine del braciere di c. $21 \mathrm{r}$ o anche il viaggio dei due innamorati evocato con la figura della suola a c. 61r. Legata a questo stesso registro dovrebbe essere anche la raffigurazione di una lucerna in forma d'anatra stretta al collo da un nastro e affiancata da un gomitolo, c. 26r, per cui cfr. l'indicazione di "firmam custodiam vitae» addotta da Casella-Pozzi, p. 166.

10. Cfr. allora i rilievi di Frederick KIEFER, «The Conflation of Fortuna and Occasio in Renaissance Thought and Iconography», Journal of Medieval and Renaissance Studies, 9, 1979, p. $1-27$.

11. Giusti i rilievi a riguardo di Alessandro RovetTA, «Il Somnium Delphili», cit., p. 64.

12. Ci si attenga dunque all'interpretazione dell'epigramma fornita da CASELLA-POZZI, op. cit., p. 165-166. Questo il testo: "Quaerenti Letum Juno sic ait: occidet amnis; / Mars cruce; Phoebus aquis. Sors rata quaeque fuit. / Arbor obumbrat aquas; ascendo; decidit ensis / Quem tuleram; casu labor et ipse super; / Pes haesit ramis, caput incidit amne. Tulique / Foemina, vir, neutrum; flumina, tela, crucem». 
stagione di straordinario credito, almeno sino all'edizione 1555 delle Symbolicarum quaestiones di Achille Bocchi. ${ }^{13}$

Se è vero dunque che questo modesto poemetto rappresenta «un significativo episodio di passaggio del mondo antiquario verso le codificazioni emblematiche» (Rossi), arriveremo a dire che anche l'intero sogno di Delfilo trova codificazione in chiave di eletta simbologia anti-naturale nella figurazione di c. 19r, dove è visibile una donna in abito succinto, monocola, coincidente con l'immagine della Speranza fallace presente all'Iconologia del Ripa: solo un presente animato da figure fantastiche resta al protagonista, a riempire il vuoto di una realtà ridotta a sterile reliquia archeologica dall'azione distruttiva del Tempo reale.

13. Si veda allora in generale per tali questioni il recente libro di Alessandra ANGELINI, Simboli e Questioni. L'eterodossia culturale di Achille Bocchi e dell'Hermathena, Bologna: Pendragon, 2003. Giova peraltro ricordare — come fa Alessandro RovETTA, «Il Somnium Delphili», cit., p. 65-67- l'importanza della presenza piacentina di Cesare Cesariano in anni coerenti all'avventura letteraria del Ceresa. 